\title{
FSRFT Based Broadband Double Matching via Passband Extremums Determination
}

\author{
R. Kopru, Member IEEE
}

\begin{abstract}
Fast simplified real frequency technique (FSRFT) is a numerical solver used to solve microwave broadband doublematching (DM) circuit design problems in a much faster and effective manner. Recently, it has been reported that an FSRFT based Matlab code can complete the design of a $n=6$ th order lowpass lumped element double matching network to match a given generator and load impedance within an optimization time of only 0.6 seconds, a 47 fold less time than that of the same design done using the classical simplified real frequency technique (SRFT). FSRFT owes this superior speed performance to the fact that it tracks only $n+1$ (system unknowns $n$ plus 1) number of passband extremum points selected from among the $n d$ number of gain data $(n d \gg n)$. This work introduces a simple numerical technique called PED (passband extremums determination technique) to be used in determination of these passband extremum points (PEs). An exemplary $n=6$ th order microwave bandpass DM circuit design using FSRFT based Matlab (of Mathworks Inc.) code and the simulation of this design via MWO (of AWR Corp.) has yielded the same circuit performance with an exact agreement. Thus, FSRFT, equipped with the PED, newly proposed hereby, might be used as a powerful solver in designing broadband circuits in many fields such as RF/microwave, radar, and communications.
\end{abstract}

Index Terms - Broadband matching, FSRFT-fast simplified real frequency technique-, lumped elements, scattering parameters.

\section{INTRODUCTION}

$\mathrm{F}$ SRFT, fast simplified real frequency technique, is a powerful RF/microwave design tool because of both its superior design completion speed and accuracy. Since its first introduction in [1], many RF/microwave filters and broadband matching network designs have been done by its author and the circuit performances yielded by FSRFT based Matlab [2] code have shown that these performances exactly agree with the results obtained from the simulations of the same circuits done via industry standard microwave packages such as MWO (Microwave Office) [3]. Design completion times are very small, even under sub-second times have been reported [1].

The major motivation in introducing of FSRFT in [1] was

R. KOPRU, is with Department of Electrical and Electronics Engineering, Isik University, Istanbul, Turkey, (e-mail: ramazan.kopru@isikun.edu.tr).

Manuscript received May 5, 2018; accepted July 23, 2018. DOI: $\underline{10.17694 / \text { bajece. } 421266}$ several difficulties faced in the optimization based design of broadband matching circuits, such as i) slow design times, ii) yielding different circuits depending on the different optimization initials. All these difficulties have been overcome with ease by using FSRFT. FSRFT is fast, gives almost always the same circuit with any randomly chosen optimization initials.

FSRFT is essentially a faster variant of the classical SRFT (simplified real frequency technique). Some important works of the classical and recent literature on broadband double matching can be counted as given in [4-9]. Since its first introduction in 1982 [10], SRFT has always found an indispensable place to itself as a very powerful solver in many broadband matching design applications such as $\mathrm{RF} /$ microwave filters, matching networks, low and high power amplifiers, LNAs (low noise amplifiers) [1, 11-20].

Previously, an example design, given in the section $\mathrm{V}$ of [1], has been done using both SRFT and FSRFT for comparison purpose. It involves the design of a $n=6$ th order lowpass equalizer, with $\varepsilon=0.333$, cut-off frequency $F_{c}=1 \mathrm{GHz}$, generator impedance $Z_{G}=\left(R_{G}+L_{G}\right) \| C_{G}$, load impedance $Z_{L}=\left(R_{L}+L_{L}\right) \| C_{L}$, with $R_{G}=12 \Omega$ $L_{G}=3 n H, C_{G}=6 p F, R_{L}=34 \Omega, L_{L}=4 n H C_{L}=8 p F$. According to Table I of [1], FSRFT completes a typical double matching design problem in far less time, 0.6 seconds (vs 29seconds) - 47 fold less time compared to those of other solvers-, since the FSRFT uses only the amplitude extremums of the gain data. Thus, FSRFT can be thought as a far superior solver in terms of speed compared to the classical SRFT. An Intel $2.20 \mathrm{GHz}$ i7 CPU with $8 \mathrm{~GB}$ RAM has been used as the computer running the experimental Matlab code [1].

Manual determination of PEs (passband extremums) and their entrance into the FSRFT based broadband double matching design code is a cumbersome process, thus, an algorithm for automatic determination of PEs is needed. Therefore, this work is devoted to present the PED (numerical passband extremums determination technique).

Organization of this paper is as follows: Section II gives a short review of broadband DM technique based on FSRFT as described in [1]. Section III introduces the newly proposed simple PED to be equipped into the FSRFT. Section IV presents a bandpass broadband DM design example solved via the FSRFT equipped with PED feature. Since a vast review of both SRFT and FSRFT has already been given in section II 
and section III of [1], those who are not familiar with SRFT and FSRFT, are recommended to consult to [1] for a much more detailed knowledge. Thus, only some summarized parts of FSRFT taken from [1] are given in the following section.

\section{REVIEW OF FAST SIMPLIFIED REAL FREQ. TECH. - FSRFT-}

In classical literature, the problem of broadband double matching is described by "designing a 2-port matching network or equalizer in an arbitrary or predefined topology constructed with lossless elements such as lumped and/or distributed inductors, capacitors, transformers and transmission lines" [1,7]. An algorithm for double matching designs in accordance with the FSRFT is given in the following algorithmic steps. Notice that the following algorithm is a version that includes the BP (bandpass) DM designs whereas the one given in [1] was only for LP (lowpass) DM designs.

\section{FSRFT Algorithm with PED for Broadband Double Matching}

\section{1) Enter the specs of the bandpass DM problem:}

- passband ripple factor, equalizer order: $\varepsilon, n$

- begin and end freqs. of the optim. range $(\mathrm{GHz}): F_{B}, F_{E}$

- lower and upper cut-off freqs. of the BP gain $(\mathrm{GHz}): F_{L}, F_{H}$

- center freq. is computed as $(\mathrm{GHz}): F_{0}=\sqrt{F_{L} F_{H}}$

2) Enter the impedance data to be matched:

- impedance data: $Z_{G}\left(\omega_{k}\right), Z_{L}\left(\omega_{k}\right), \omega_{k}=2 \pi F_{k}$.

- frequency data: $F_{k}(\mathrm{GHz}), k=1,2, \ldots n d$.

3) Generate normalized frequency and impedance data:

- freq. normalization factor: $\Omega=2 \pi F_{0}$, $(\mathrm{Grad} / \mathrm{s})$

- norm. freq: $f_{b}=F_{B} / \Omega, f_{l}=F_{L} / \Omega, f_{h}=F_{H} / \Omega, f_{e}=F_{E} / \Omega$

- normalized center freq.: $\omega_{0}=2 \pi\left(F_{0} / \Omega\right)=1(\mathrm{rad} / \mathrm{s})$

- impedance norm. factor: $R_{0}=50 \Omega$

- norm. imp.: $z_{G}\left(\omega_{k}\right)=Z_{G}\left(\omega_{k}\right) / \mathrm{R}_{0}, z_{L}\left(\omega_{k}\right)=Z_{L}\left(\omega_{k}\right) / \mathrm{R}_{0}$.

- norm. freq.: $f_{k+1}=f_{k}+\Delta f, \Delta f=\left(f_{2}-f_{1}\right) /(n d-1)$.

- norm. complex fr.: $p_{k}=j \omega_{k}, \omega_{k}=2 \pi f_{k}, k=1,2, \ldots n d$

4) Generate target gain func. $T_{t}$ to be tracked by the optimization:

$$
T_{t}\left(\omega_{k}\right)=\frac{1}{1+\varepsilon^{2} T_{n}\left(p_{k}\right)^{2}}
$$

where $T_{n}\left(p_{k}\right)$ is an $n$th order Chebyshev function.

$$
\begin{aligned}
& \text { 5) Construct "initial target data, ITD": } \\
& \operatorname{ITD}(k)=\left\{\omega_{k}, T_{t}\left(\omega_{k}\right), z_{G}\left(\omega_{k}\right), z_{L}\left(\omega_{k}\right)\right\}, k=1,2, \ldots n d
\end{aligned}
$$

6) Form a "selected target data STD":

STD is constructed from passband extremums (PEs) of ITD (initial target data) using the PED (passband extremums determination technique) proposed in this work (PED is explained in detail in section III)

$$
\operatorname{STD}(i)=\left\{\omega_{i}, T_{t}\left(\omega_{i}\right), z_{G}\left(\omega_{i}\right), z_{L}\left(\omega_{i}\right)\right\}, i=1,2, \ldots, n+1
$$

Or, in terms of PEs, ,STD $(i)=\left\{P E(i), z_{G}\left(\omega_{k}\right), z_{L}\left(\omega_{k}\right)\right\}$.

7) Run the "constraint optimization" code

With the initial 'unknowns' vector $x_{0} \leftarrow h$, call the optimization function $O p t_{-}$FSRFT $(x)$,

\section{$\left[x, f_{\text {val }}\right]=$ cons_optim('Opt_FSRFT' $\left., x_{0}, l b, u b\right)$}

where $l b$ and $u b$ are lower and upper boundaries of the constraint optimization that can be set to sufficiently small values so as to make the optimization as fastly converging as possible.

\section{8) Optimization function: Opt_FSRFT(x):}

- $h_{i} \leftarrow x_{i},(i=1,2, \ldots, n+1)$.

- Set the polynomial $f=\left[\begin{array}{llllll}0 & 0 & \ldots & 1 & \ldots & 0\end{array}\right]$, i.e. midterm is 1 .

$$
f(p)=f_{1} p^{n}+f_{2} p^{n-1}+. .+f_{n d c} p^{n d c}+. . f_{n+1}
$$

where all $f_{i}=0$,except $f_{\text {ndc }}=1$. Note that $n d c$ is the number of DC transmission zeros and generally chosen as $n d c=n / 2$ to obtain a symmetrical bandpass gain shape.

- Calculate the even polynomials $H, F, G$ by

$$
\begin{gathered}
H=h h^{\dagger} \\
F=f f^{\dagger} \\
G=H+F \\
G=g g^{\dagger}
\end{gathered}
$$

where $\dagger$ sign denotes "paraconjugate" of any polynomial $x(p)$, i.e. $x^{\dagger}=x(-p)$. The polynomial $g$ must be a strictly Hurwitz polynomial for obtaining realizable networks and can be obtained from even polynomial $G$ by

$$
g=\sqrt{G(1)} \operatorname{poly}(\operatorname{LHProots}(G))
$$

where $G(1)$ is the $1^{\text {st }}$ coefficient of the polynomial $G$ and poly(LHProots $(G))$ Matlab command represents a polynomial constructed of left half plane roots selected among the roots of the polynomial $G . g$ is written in a form of $[1,7]$,

$$
g(p)=g_{1} p^{n}+g_{2} p^{n-1}+\cdots+g_{n} p+g_{n+1}
$$

- Compute scattering matrix $S$ in Belevitch form by

$$
S=\left[\begin{array}{ll}
S_{11} & S_{12} \\
S_{21} & S_{22}
\end{array}\right]=\frac{1}{g}\left[\begin{array}{cc}
h & \mu f^{\dagger} \\
f & -\mu h^{\dagger}
\end{array}\right]
$$

where $\mu$ is unimodular constant $\left(\mu=f^{\dagger} / f=\mp 1\right)[1,7]$. In SRFT formalism, the ultimate aim of designing an equalizer is to be able to optimize the coefficients of the numerator polynomial $h(p)$ of the input reflection function

$$
S_{11}(p)=h(p) / g(p)
$$

- Compute the gain $T\left(\omega_{i}\right)$ via (10) or (11) as follows $[1,7]$,

$$
\begin{aligned}
& T_{G}=\left(1-\left|S_{G}\right|^{2}\right) \frac{\left|S_{21}\right|^{2}\left(1-\left|S_{L}\right|^{2}\right)}{\left|1-S_{11} S_{G}\right|^{2}\left|1-S_{2} S_{L}\right|^{2}} \\
& T_{L}=\left(1-\left|S_{L}\right|^{2}\right) \frac{\left|S_{12}\right|^{2}\left(1-\left|S_{G}\right|^{2}\right)}{\left|1-S_{22} S_{L}\right|^{2}\left|1-S_{1} S_{G}\right|^{2}}
\end{aligned}
$$


obviously,

$$
T=T_{G}=T_{L}
$$

where

$$
S_{1}=S_{11}+\frac{S_{12} S_{21} S_{L}}{1-S_{22} S_{L}}, \quad S_{2}=S_{22}+\frac{S_{12} S_{21} S_{G}}{1-S_{11} S_{G}}
$$

and

$$
S_{G}=\frac{Z_{G}-1}{Z_{G}+1}, \quad S_{L}=\frac{Z_{L}-1}{Z_{L}+1}
$$

where $S_{k m},(k=1,2 ; m=1,2)$ are the elements of the unitnormalized scattering matrix $[S]$ of the equalizer E. $S_{G}$ and $S_{L}$ are generator and load reflectances expressed in terms of generator and load impedances $Z_{G}$ and $Z_{L}[1,7]$. $S_{1}$ and $S_{2}$ are input and output reflectance functions, respectively, seen at the related port of the matching network when the other port is terminated with $Z_{L}$ and $Z_{G}$, respectively $[1,21]$. A much simpler and handy impedance based gain formulae which are equivalent to the reflectance based gain formulae given in (10) and (11) can also be used to calculate the gain such that [21]

$$
\begin{aligned}
& T_{G}=\frac{4 R_{1} R_{G}}{\left|Z_{1}+Z_{G}\right|^{2}}=\frac{4 R_{1} R_{G}}{\left(R_{1}+R_{G}\right)^{2}+\left(X_{1}+X_{G}\right)^{2}} \\
& T_{L}=\frac{4 R_{2} R_{L}}{\left|Z_{2}+Z_{L}\right|^{2}}=\frac{4 R_{2} R_{L}}{\left(R_{2}+R_{L}\right)^{2}+\left(X_{2}+X_{L}\right)^{2}}
\end{aligned}
$$

- Compute error func.: $d_{i}=T\left(\omega_{i}\right)-T_{t}\left(\omega_{i}\right)$.

- optimization ends with success,

$$
\text { iff }\left\{\text { sse }=\sum_{i=1}^{i_{\max }} d_{i}^{2}\right\} \rightarrow \delta \sim 0,
$$

yielding optimized polynomial $h$ such that $h \leftarrow x_{\text {opt }}$. sse is known as 'sum of squared errors' [1].

\section{9) Network Synthesis and Gain Plotting:}

- Make the final design calculations repeating (2) to (16) using the optimized polynomial $h \leftarrow x_{\text {opt }}$.

- Compute the driving point input impedance $Z_{11}(p)$ of the equalizer by $[1,7]$

$$
Z_{11}(p)=\frac{a(p)}{b(p)}=\frac{g(p)+h(p)}{g(p)-h(p)}
$$

where polynomials $a$ and $b$ are given in the form $[1,7]$

$$
\begin{gathered}
a(p)=a_{1} p^{m}+a_{2} p^{m-1}+\cdots+a_{m} p+a_{m+1} \\
b(p)=a_{1} p^{n}+a_{2} p^{n-1}+\cdots+a_{n} p+a_{n+1}
\end{gathered}
$$

- Polynomials $a(p)$ and $b(p)$ are entered into a Matlab based high precision synthesis package [2224] which then extracts the schematic with element values of the designed equalizer.

- Plot $T(\omega)$ vs $T_{t}(\omega)$ to check if the gain performance
$T$ of the designed equalizer agree well with the desired target gain $T_{t}$ performance.

The equalizer designed in the Matlab environment [2] can be simulated in a commercial microwave package such as MWO [3].

\section{PED - PASSBAND EXTREMUMS DETERMINATION TECH.}

This section introduces the proposed PED (numerical passband extremums determination technique) which is embedded into the FSRFT. PED is a simple technique to find the passband extremums (PEs) of a given target gain function $T_{t}(\omega)$ for the DM system seen in Fig.1. In FSRFT, these $n+1$ number of predetermined PEs are then tracked by the optimization algorithm to optimize the $n+1$ number of coefficients $h_{i}(i=1,2, \ldots, n+1)$ of the polynomial $h(p)$ given in (2).

Fig. 2 shows an example target gain function $T_{t}(\omega)$ with $n d$ number of data ( $n d \gg n$ ) generated by an $n$th order Chebyshev approximation using (1). Use of this target gain function in broadband equalizer design would elapse great time due to the fact that the optimization algorithm must track an $n d$ number of data, $n d \gg n$, which is much greater than the order $n$ of the equalizer to be designed. However, with FSRFT, the optimization algorithm has to track only $n+1$ number of data which are predetermined at the exact positions or vicinity of the PEs of the target gain function. Tracking PEs

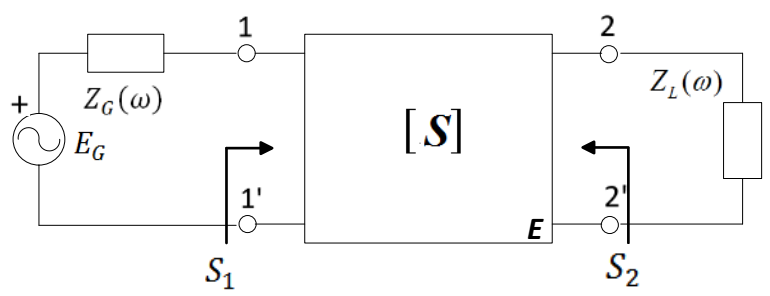

Fig.1. Broadband double matching problem.

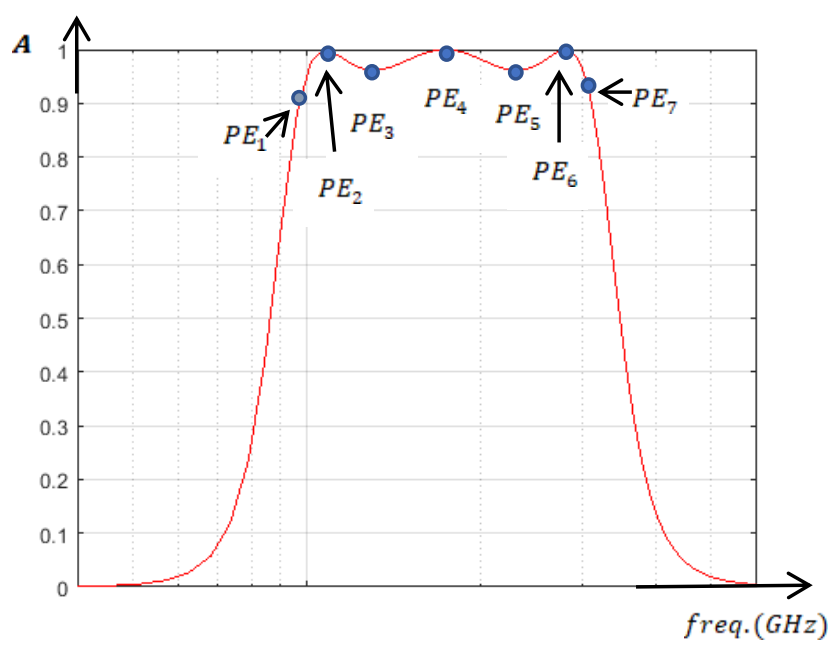

Fig.2. PED-passband extremums determination technique-: Chebyshev approximated $n$th order target gain function $T_{t}(\omega)$ is plotted (solid red line) with $n d$ number of gain amplitudes $\boldsymbol{A}$ vs.freq. (nd $\gg n)$. $n+1$ number of passband extremums PEs (blue dots) are determined at passband extremum points over the target gain function $T_{t}(\omega)$. 
in the optimization process is very advantageous to reduce the optimization time because the PEs are already at the exact points where the solution of the system resides. "Therefore, selection of $n+1$ number of frequency locations $\omega_{i}(i=1,2, \ldots, n+1)$ where the passband extremums $T_{t}\left(\omega_{i}\right)$ occur is naturally the wisest selection having a potential to hit the solution $h_{\text {opt }} \leftarrow x_{\text {opt }}$ as quick as possible" [1].

Passband extremums determination algorithm given below is a numerical method that uses a given target gain function $T_{t}(\omega)$ so that a pair of frequency and gain amplitude at each passband extremum of $T_{t}$ function is calculated such that $P E_{i}=\left(\omega_{i}, T_{t}\left(\omega_{i}\right)\right), i=1,2, \ldots, n+1$. In the literature, available equations [7] can be used to find the passband extremums analytically for an $n$th order Chebysev function, however the method presented here is a numerical one that might be used for a gain function with any arbitrary shape.

\section{PED -Passband Extremums Determination-Algorithm}

STEP 1. Enter the given target gain function $T_{t}$ as a pair of frequency and gain amplitude at each $k$, i.e. $T_{t}(k)=T_{t}\left(\omega_{k}, A_{k}\right), k=1,2, \ldots, n d$. If the number of gain data $n d$ is not sufficiently large (data number is close to the order $n$ of the equalizer to be designed, i.e. $n d \cong n$, or less ), apply an interpolation to obtain a gain function $T_{t}(k)$ with sufficiently large number of data such that $n d \gg n$, for instance $n d \geq 15(n+1)$.

STEP 2. The first passband extremum $P E(1)$ is determined by the designer by examining the $T_{t}$ gain plot (Fig.2) and it is chosen estimately at a frequency $\omega_{k}$ around where the passband begins as seen in Fig.2. Thus, $P E(1)$ is entered to the code as $P E(i)=T_{t}(k)$ for $i=1$, or equivalently, $\operatorname{PE}\left(\omega_{i}, A_{i}\right)=T_{t}\left(\omega_{k}, A_{k}\right)$ again for $i=1$.

The frequency index $k$ is increased by 1 , i.e. $k \leftarrow k+1$.

STEP 3. A 1st local slope is calculated as $m_{1}=A_{k}-A_{k-1}$ The frequency index $k$ is increased by 1 , i.e. $k \leftarrow k+1$. A 2nd local slope is calculated as $m_{2}=A_{k}-A_{k-1}$ The frequency index $k$ is increased by 1 , i.e. $k \leftarrow k+1$.

STEP 4. If the product of two slopes satisfies $m_{12}=m_{1} m_{2}>0$, this means that two successive slopes have the same direction indicating that this frequency point $\omega_{k}$ can not be a new extremum (PE). Then, goto STEP 3.

Otherwise, if $m_{12}=m_{1} m_{2}<0$, this means that two successive slopes have opposite direction indicating that a new extremum (PE) is located at this frequency point $\omega_{k}$. Then, increase $i$ and assign $P E(i)=T_{t}(k)$.
If $i<n+1$ Goto STEP 3, Otherwise goto STEP 5 .

STEP 5. All passband extremums $P E(i), i=1,2, \ldots, n+1$ have now been determined via the PED algorithm given in the above steps. Thus, continue the design from the Step 6 of the FSRFT Algorithm given in section III.

MATLAB CODE: A Matlab function developed using the PED algorithm given above is listed as follows:

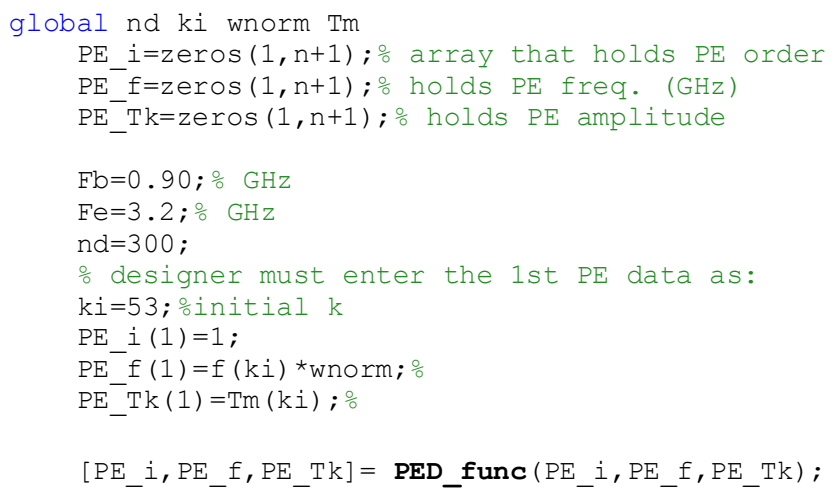

\section{DESIGN EXAMPLE}

A bandpass double matching (DM) problem is given in this section and solved via FSRFT based on the proposed PED technique. A broadband design is to be done involving an $n=6$ th order bandpass Chebyshev approximated lumped element equalizer, with passband ripple factor $\varepsilon=0.20$, lower and upper cut-off frequencies of $F_{L}=1 \mathrm{GHz}$, $F_{H}=3 \mathrm{GHz}$, generator impedance $Z_{G}=\left(R_{G}+L_{G}\right) \| C_{G}$, and load impedance $Z_{L}=\left(R_{L}+L_{L}\right) \| C_{L}$, built of $R_{G}=40 \Omega$, $L_{G}=0.8 n H, C_{G}=1 \mathrm{pF}, R_{L}=30 \Omega, L_{L}=1.2 \mathrm{nH}, C_{L}=0.6 \mathrm{pF}$ Solution via FSRFT with PED:

First, the optimization frequency range can be chosen for instance varying from $F_{B}=0.9 \mathrm{GHz}$ upto $F_{E}=3.2 \mathrm{GHz}$. Using FSRFT algorithm given in section III, initial target data 


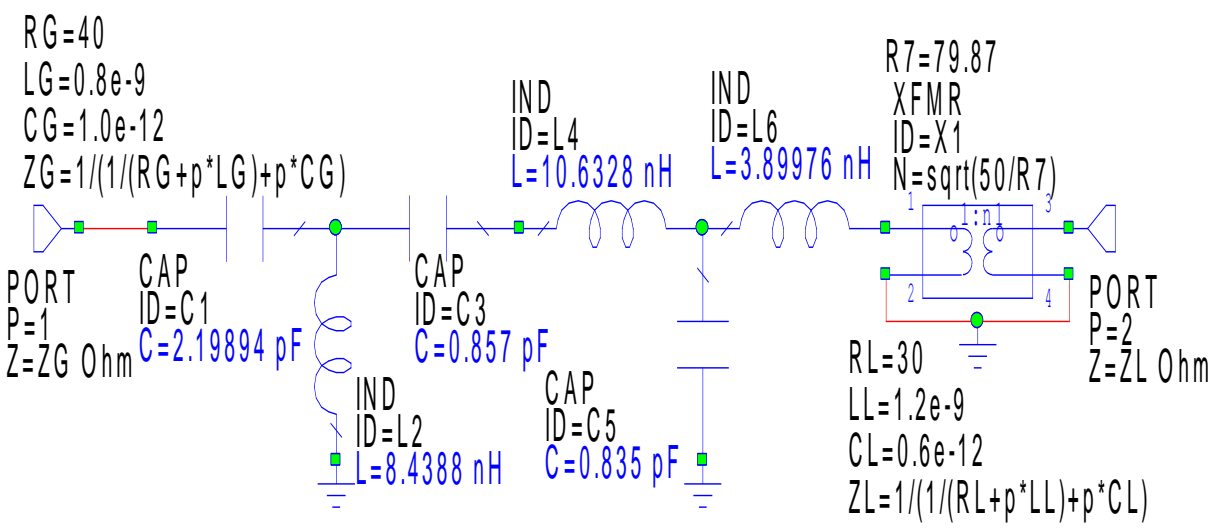

a)

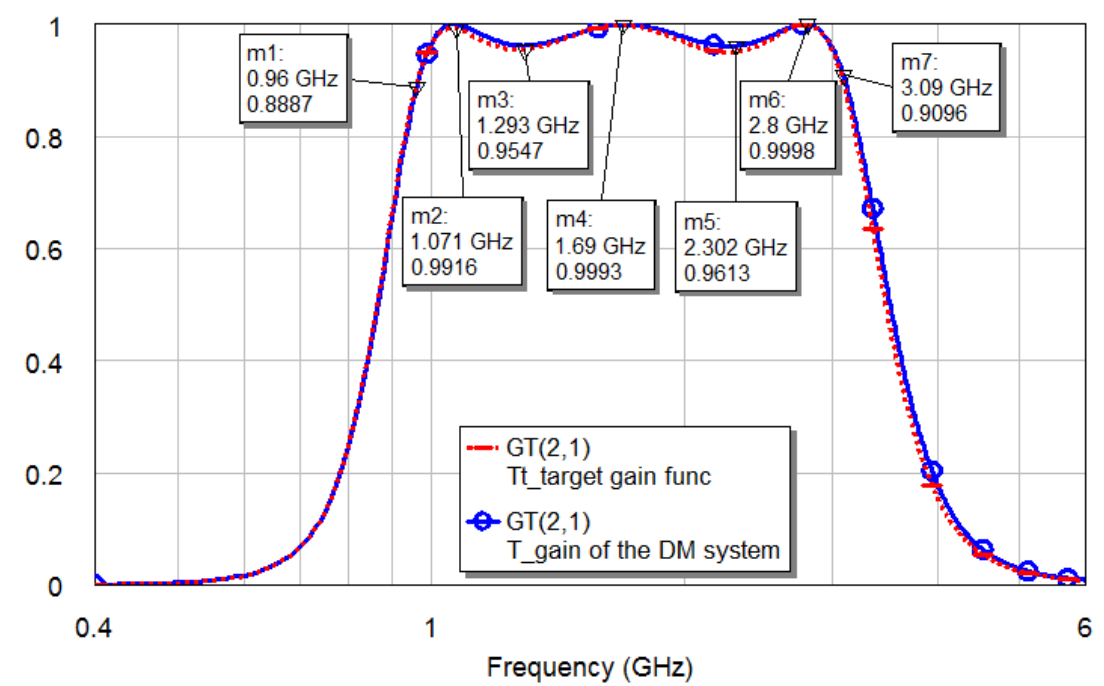

b)

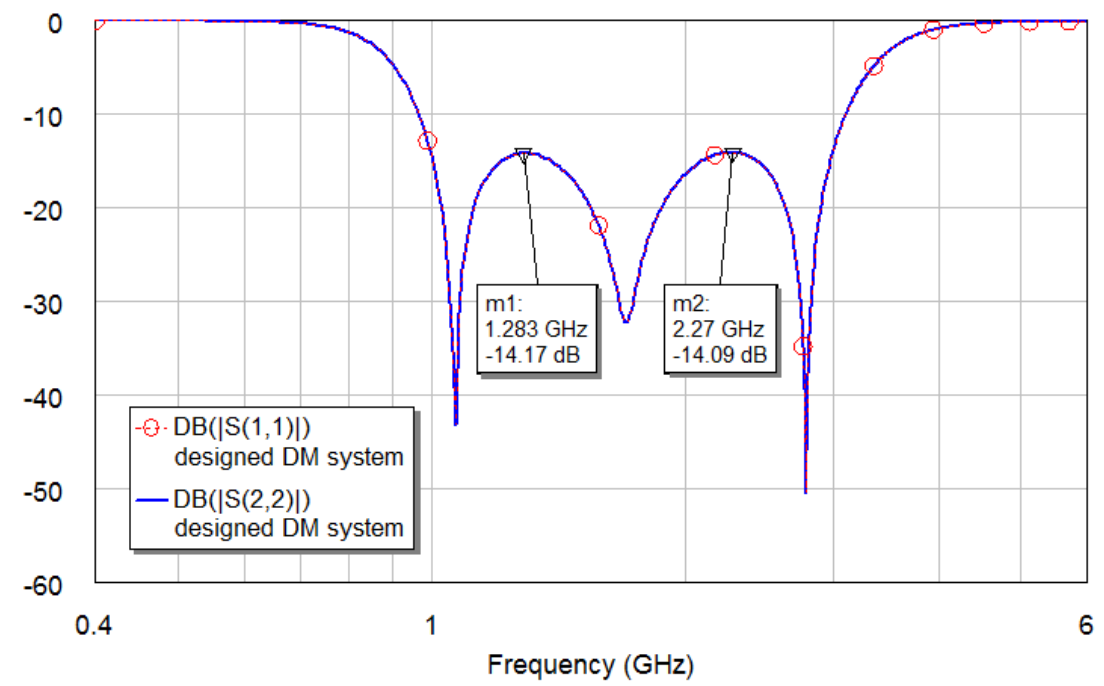

Fig.3. MWO [3] Simulation results of the example DM system design done via the Matlab code: a) overall circuit schematic of the DM system designed via the Matlab code is redrawn in MWO. b) gain performance of the DM system.

c) input/output reflectance performance of the DM system. 
set ITD is generated for the whole range of optimization frequencies $\left[F_{B}, F_{E}\right]=\left[\begin{array}{ll}0.9, & 3.2\end{array}\right] \mathrm{GHz}, \quad$ for example, $n d=300$ freq. points, i.e. $\operatorname{ITD}(k)=\left\{\omega_{k}, T_{t}\left(\omega_{k}\right), z_{G}\left(\omega_{k}\right), z_{L}(\right.$ $\left.\left.\omega_{k}\right)\right\}, k=1,2, \ldots, k_{\max }=n d=300$. Therefore, the $P E D$ Algorithm given in section III, determines the indices of the passband extremums occurring at the indices of $k_{\text {ext }}=\{9,23,53,109,183,249,286\}$ whose element number is the equalizer order $n$ plus 1 , i.e. $n+1=7$. Then the passband extremums are determined by the Matlab code $P E D \_f u n c$ as $P E(i)=T_{t}(k), P E=\left\{P E_{1}, P E_{2}, P E_{3}, P E_{4}, P E_{5}\right.$, $\left.P E_{6}, P E_{7}\right\}=\left\{T_{t}(9), T_{t}(23), T_{t}(53), T_{t}(109), T_{t}(183), T_{t}(249)\right.$, $\left.T_{t}(286)\right\}$, where $P E(i)=P E\left(f_{i}, A_{i}\right)=\{(0.960,0.882),(1.07$, $1.00),(1.296,0.962),(1.688,0.999),(2.304,0.962),(2.808$, $1.000),(3.088,0.911)\}$, where all $f_{\mathrm{i}}$ frequencies are given in $\mathrm{GHz}$. It is better to choose $n d$ as large as possible, for instance $n d=300$ to obtain accurate PE locations. Table I shows the determined PE list for $n d=300$. The optimization range in determining the PEs of Table $\mathrm{I}$ has been chosen as $\left[F_{B}, F_{E}\right]=[0.90,3.20] \mathrm{GHz}$. The selected target data set $\operatorname{STD}(i)$ is then constructed by one to one mapping from $\operatorname{ITD}(k)$ to $S T D(i)$ yielding $\operatorname{STD}(i)=\left\{P E(i), z_{G}\left(\omega_{k}\right), z_{L}\left(\omega_{k}\right)\right\}$.

TABLE I

PEs DETERMINED BY MATLAB FUNCTION PED_FUNC()

\begin{tabular}{l|rl}
$P E_{s}$ & $f_{i}(\mathrm{GHz})$ & amplitude $(A)$ \\
\hline$P E_{1}$ & 0.960 & 0.884 \\
$P E_{2}$ & 1.071 & 0.999 \\
$P E_{3}$ & 1.303 & 0.962 \\
$P E_{4}$ & 1.732 & 0.999 \\
$P E_{5}$ & 2.303 & 0.962 \\
$P E_{6}$ & 2.803 & 1.000 \\
$P E_{7}$ & 3.088 & 0.911
\end{tabular}

Upon a succesful optimization, the code yields the optimized polynomial as $h(p)=0.352 p^{6}+0.322 p^{5}+1.216 p^{4}$ $+0.540 p^{3}+1.216 p^{2}+0.109 p+0.386$ with the polynomial $g(p)=0.352 p^{6}+1.009 p^{5}+2.513 p^{4}+3.044 p^{3}+2.574 p^{2}$ $+1.029 p+0.386$ and $f(p)=p^{3}$. The designer having the optimized polynomial $h(p)$ given above, can reconstruct the equalizer circuit using only this polynomial $h(p)$ without reexecuting the optimization code at all but following only the remaining steps of the FSRFT Algorithm given in section II. Overall circuit schematic of the DM system (with the designed equalizer between the ports) is seen in Fig.3.a. In Fig.3.b and c, gain and input/output reflectance performance of the designed DM system are shown. Fig.3.b shows clearly that the gain performance of the designed DM system is in almost excellent agreement with that of the desired target gain function $T_{t}$. Notice that the measurement points marked by $\mathrm{m}_{1}$, $\mathrm{m}_{2}, \ldots, \mathrm{m}_{7}$ are the PEs of the system. Fig.3.c shows also that the input/output reflectance performance of the designed DM system is very well, around $-14.1 \mathrm{~dB}$ pretty below $-10 \mathrm{~dB}$ along the operating band.

FSRFT completes the solution of this broadband bandpass DM design problem within as low as only 0.58 seconds using only $n+1=7$ number of PEs. The same computer, an Intel 2.20 GHz i7 CPU with 8 GB RAM, that has been used in the previous work of [1] has been used as the computer running the experimental Matlab code here.

\section{CONCLUSION}

The proposed PED is a simple but very effective technique in determining the PEs which eases the design process of broadband DM system tremendously. The work presented here gives also the design method of broadband bandpass matching networks which are much more needed in wideband microwave amplifiers compared to their lowpass counterparts. Thus, need for bandpass designs using FSRFT has also been met with this work. With the addition of proposed PED feature here, FSRFT might be a good candidate to be a fast and effective design tool utilizable in broadband $\mathrm{RF} /$ microwave area.

\section{REFERENCES}

[1] R. Kopru, "FSRFT - fast simplified real frequency technique via selective target data approach for broadband double matching", IEEE Transactions on circuits and systems-II: Express briefs, Vol. 64, No. 2, february 2017, pp.141-145.

[2] Matlab, https://www.mathworks.com Mathworks Inc., MA., USA, retrieved on april 8, 2018.

[3] MWO www.awrcorp.com, AWR Corp, retrieved on april 8, 2018.

[4] H. J. Carlin, "A new approach to gain-bandwidth problems," IEEE Trans. Circuits Syst., vol. 24, no. 4, pp. 170-175, Apr. 1977.

[5] B. S. Yarman, "Broadband matching a complex generator to a complex load," Ph.D. dissertation, ECE Dept. Cornell Univ., NY, USA, 1982.

[6] B. S. Yarman, Design of Ultra Wideband Antenna Matching Networks Via Simplified Real Frequency Techniques, Netherlands: Springer, 2008, pp. 183-225.

[7] B. S. Yarman, Design of Ultra Wideband Power Transfer Networks, 1st ed., Chichester, UK.: John Wiley \& Sons Ltd., 2010.

[8] W. K. Chen, Broadband Matching:Theory and Implementations, 3rd ed., World Scientific, ISBN: 978-9971-5-0219-5, Nov. 1998.

[9] Y. S. Zhu, W. K. Chen, Computer-Aided Design of Communication Networks, World Scientific, ISBN: 978-981-02-2351-9, April 2000.

[10] B. S. Yarman and H. J. Carlin, "A simplified real frequency technique applied to broad-band multistage microwave amplifiers," IEEE Trans. Microw. Theory Techn., vol. 30, no. 12, pp. 2216-2222, Dec. 1982.

[11] L. Zhu, B. Wu, and C. Sheng, "Real frequency technique applied to synthesis of broad-band matching networks with arbitrary nonuniform losses for MMICs," IEEE Trans. Microw. Theory Techn., vol. 36, no. 12, pp. 1614-1620, Dec. 1988.

[12] P. Jarry and A. Perennec, "Optimization of gain and VSWR in multistage microwave amplifier using real frequency method," in Proc. ECCTD, Paris, FR., 1987, pp. 203-208.

[13] R. Kopru, H. Kuntman, and B. S. Yarman, "On numerical design technique of wideband microwave amplifiers based on $\mathrm{GaN}$ small-signal model," Analog Integr. Circ. Sig. Procc., vol. 81, no. 1, pp. 71-87, Jul. 2014.

[14] G. Sun and R. H. Jansen, "Broadband doherty power amplifier via real frequency technique," IEEE Trans. Microw. Theory Techn., vol. 60, no. 1, pp. 99-111, Jan. 2012.

[15] N. Tuffy, L. Guan, A. Zhu, and T. J. Brazil, "A simplified broadband design methodology for linearized high-efficiency continuous class-F power amplifiers," IEEE Trans. Microw. Theory Techn., vol. 60, no.6, pp. 1952-1963, Jun. 2012.

[16] Y. Sun and X. Zhu, "Broadband continuous class $-\mathrm{F}^{-1}$ amplifier with modified harmonic-controlled network for advanced long term evolution application," IEEE Microw.Compon. Lett., vol. 25, no.4, pp. 250-252, Jun. 2015.

[17] L. Ma, J. Zhou, and W. Huang, "A broadband highly efficient harmonictuned power amplifier exploiting compact matching network," IEEE Microw.Compon. Lett., vol. 25, no.11, pp. 250-252, Nov. 2015.

[18] R. Kopru, H. Kuntman and B. S. Yarman, "Novel approach to design ultra wideband microwave amplifiers: normalized gain function method". Vol. 22, No. 3, pp. 672-686, September 2013, Radioengineering. 
[19] R. Kopru, S. Kılınç, C. Aydın, D. C. Atilla, C. Karakuş, B. S. Yarman, "Ultra wideband matching network design for a V-shaped square planar monopole antenna", International Journal of Microwave and Wireless Technologies, Cambridge Univ. Press, 2014, volume 6, issue 06, pp. 555-564.

[20] R. Kopru, S. Kilinc, A. Aksen, and B. S. Yarman, "Design and implementation of wideband microwave amplifiers based on normalized gain function", BenMAS2014, 2014 IEEE Benjamin Franklin Symposium on Microwave and Antenna Sub-Systems, Radar, Telecommunications, and Biomedical Applications, September 27, 2014, Philadelphia, Pennsylvania, USA.

[21] M. Sengul, "Design of practical broadband matching networks with lumped elements," IEEE Trans. Circuits Syst. II, Exp. Briefs, vol. 60, no. 9, pp. 552-556, Sep. 2013.

[22] A. K1lınç and B. S. Yarman, "High precision LC ladder synthesis part I: Lowpass ladder synthesis via parametric approach," IEEE Trans. Circuits Syst. I, Reg. Papers, vol. 60, no. 8, pp. 2074-2083, Aug. 2013.

[23] B. S. Yarman, and A. Kılınç, "High precision LC ladder synthesis part II: Immitance synthesis with transmission zeros at DC and infinity," IEEE Trans. Circuits Syst. I, Reg. Papers, vol. 60, no. 10, pp. 27192729, Oct. 2013.

[24] B. S. Yarman, A. Aksen, R. Kopru, N. Kumar, C. Aydin, D. C. Atilla, P. Chacko, "Computer aided Darlington synthesis of an all purpose immittance function", IU-JEEE Istanbul University Journal of Electrical and Electronics Engineering, Vol. 16, No. 1, pp. 2027-2037, 2016.

\section{BIOGRAPHIES}

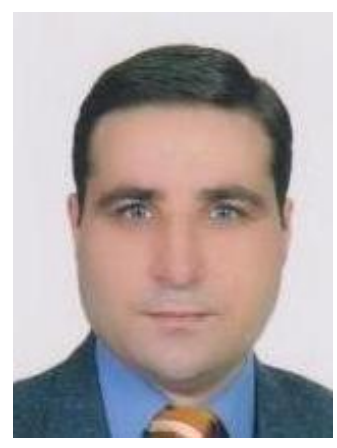

RAMAZAN KOPRU received

B.Sc. degree from Yildiz Technical University in 1991, M.Sc. and Ph.D. degrees from Istanbul Technical University, Turkey, in 1994 and 2013, respectively, all from electronics and communications engineering. In the past, he has worked as a research and development engineer in the fields of embedded systems, wireless systems, power electronics and

defense electronics. Since 2014, he has been an Assistant Professor with the Electrical and Electronics Engineering Department, Işık University, Istanbul, Turkey. He is the author/co-author of 41 articles published in international/national peer-reviewed journals and conferences. $\mathrm{He}$ is also the referee of many papers for the international peer-reviewed journals and conferences. His main research areas are military spec low noise wideband analog design for laser signal detection and post processing, wideband microwave low power and power amplifier design using real frequency techniques, network synthesis with lumped and distributed elements, fast circuit design methods for $\mathrm{RF} /$ microwave broadband matching using FSRFT, high voltage techniques, optimization, power system analysis, power electronics, embedded systems, supercapacitors. 